Research Square

\title{
Burden of Meningococcal Disease in the United Kingdom During the Period 2008 to 2017 - A Secondary Database Analysis
}

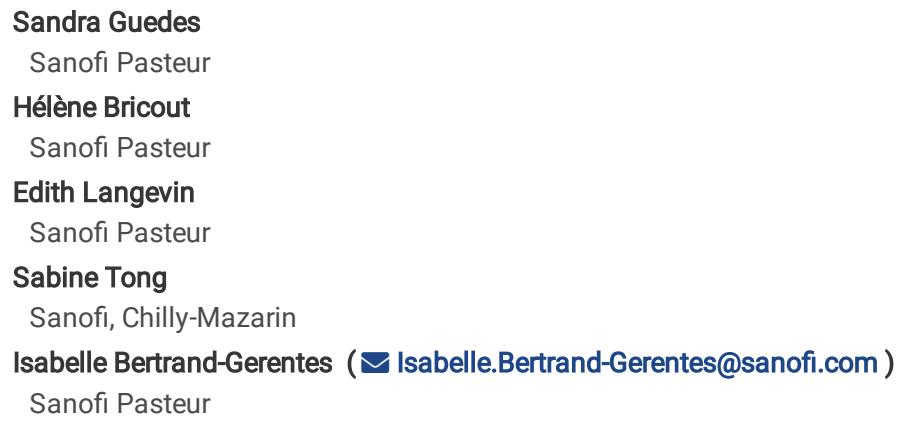




\section{Abstract}

\section{Background}

Invasive meningococcal disease (IMD) causes high fatality in untreated patients alongside long-term sequelae in $20 \%$ survivors. For a comprehensive assessment of disease burden, an analysis of these sequelae is required. This study aims to investigate the burden of disease between 2008 and 2017 including a description of the sequelae, through the analysis of data collected from the UK Clinical Practice Research Datalink (CPRD) linked with data from the Hospital Episode Statistics (HES), and Office for National Statistics (ONS) mortality registry data.

\section{Methods}

This was a 10-year retrospective observational cohort study designed to describe the incidence, case-fatality rate (CFR) and occurrence of sequelae due to meningococcal disease, in the UK between 2007 and 2017 using data from the UK CPRD-HES-ONS. Cases were identified and matched on age, gender, date of diagnosis of IMD and followed-up-time with a control group without IMD. Demographics, clinical characteristics, mortality, and IMD-related sequelae were examined for IMD cases and compared with matched controls for a more comprehensive burden of disease assessment.

\section{Results}

The study analysed 640 IMD patients with majority of the cases diagnosed (76.9\%) in a hospital setting. Age-group analysis showed a decrease in the incidence rate of IMD in patients aged $<1$ year $(30.4-7.5 \%)$ and an increase in those $>50$ years $(10.4-27.8 \%)$. CFR was slightly higher among females, toddlers, and adults $>50$ years. No significant change in CFR was observed over study period. Case-control study showed a higher number of IMD sequelae among cases compared to age- and gender-matched controls, especially in those $\geq 50$ years.

\section{Conclusion}

The study showed that, despite a relatively low incidence rate, IMD is responsible for a high CFR, namely in older age groups and by a high number of IMD sequelae. The study showed that leveraging data from existing databases can be used to complement surveillance data in truly assessing the burden of IMD. Despite the availability of routine vaccination programs, IMD still poses a significant burden in the healthcare system of the UK. Optimization of vaccination programs may be required to reduce the disease burden.

\section{Background}

Invasive meningococcal disease (IMD), caused by Gram-negative bacterium Neisseria meningitidis, is a potentially fatal disease. Nearly $8 \%$ to $15 \%$ of patients with IMD die even when the disease is diagnosed early, and adequate treatment is started. If untreated, IMD is fatal in $50 \%$ of patients and can cause longterm sequelae including brain damage, hearing loss, or disability in up to $20 \%$ of survivors [1]. The clinical presentation of IMD is diverse with meningitis and septicemia being the most common modes of presentation. The severity of manifestations ranges from bacteraemia, associated with mild, non-specific symptoms, to fulminant sepsis with multiorgan failure and death. Localised infections (such as conjunctivitis or septic arthritis) as well as chronic disease may be the sole clinical manifestations but can lead to disseminated fulminant disease [2]. Twelve serogroups of $N$. meningitidis have been identified, with six serogroups - A, B, C, W, X, and $\mathrm{Y}$ - being responsible for virtually all invasive disease [2].

The epidemiology of IMD is dynamic, with different geographical distributions and varying incidence of $N$. meningitidis serogroups and the emergence of new strain variants [3]. Around 1.2 million people are estimated to be diagnosed with IMD per year, with nearly 135,000 case fatalities worldwide [4]. Although IMD affects individuals of all ages, the highest incidence occurs in young children, with a second disease peak among adolescents and young adults [5, 6]. The incidence is also high in the elderly population, the age group with the highest case fatality rate (CFR) [7-10].

According to the Global Disability-Adjusted Life Years (DALY) estimation, the burden of all-age meningitis from all causes was 20.4 million DALY (range: 17.823.4) in 2017 [11]. In younger ages, meningococcal meningitis and other bacterial meningitis are the predominant causes of new cases and deaths. Meningitis and meningococcal meningitis also causes a high burden in the elderly population, with increasing levels of incidence, mortality, and Years of Life lived with Disability (YLD) rates [12].

The most effective approach to prevent IMD is through vaccination [13]. Although the United Kingdom (UK) became the first country in the world to routinely vaccinate against serogroups $B$ and $C$, the incidence of meningococcal disease across all age groups is still relevant $[14,15]$. Meningococcal serogroup $C$ (Men-C) conjugated vaccine was introduced in the UK in 1999, and the cases of IMD fell dramatically by over $90 \%$ in immunized age groups and indirectly, by two-thirds in other age groups due to reduced carriage and exposure. The emergence of serogroup $B$ and serogroup W led to the introduction of meningococcal serogroup B (MenB) vaccine in infant immunization schedule in 2015 and the replacement of MenC with meningococcal (Men) ACWY vaccine in adolescents, respectively [16].

This study aims to investigate the burden of meningococcal disease in the UK during the period between 2007 and 2017 through the analysis of data collected from the UK Clinical Practice Research Datalink (CPRD) linked with data from the Hospital Episode Statistics (HES), and Office for National Statistics (ONS) mortality registry, including a description of the sequelae following meningitis disease for a more comprehensive burden of disease assessment.

\section{Methods}

\section{Study design}


This was a 10-year retrospective observational cohort study designed to describe the incidence and the Case-Fatality Rate (CFR) due to meningococcal disease, as well as the occurrence of sequelae in the UK between 2007 and 2017 using data from Clinical Practice Research Datalink (CPRD) GOLD, linked with data from the Hospital Episode Statistics (HES), and Office for National Statistics (ONS) mortality registry data. The CPRD is an ongoing primary care database of anonymised medical records from general practitioners, in the UK. Patients with an event of meningococcal disease were identified between 2008 and 2017 and were individually matched with up to four randomly selected controls based on age, gender, region, date of meningococcal disease diagnosis and follow-up duration. Index date was defined as the first meningococcal disease episode that occurred between 2008 and 2017 . Controls were used for only the second part of the study, i.e., for the comparison of the occurrence of sequelae between cases and controls. A baseline period of 12 -month of available data pre-index date and was required as an inclusion criterion for all patients aged $\geq 1$ year. Follow-up period was defined by all reliable data available after index date until the earliest of the following events: date of last collection, date of transfer out of the general practitioner (GP) practice, or the date of death (Fig. 1).

\section{Study population/Data source}

Assessment of incidence and CFR of meningococcal disease included all patient records from January 1, 2008 to December 31,2017 with a Read code (supplementary appendix) for meningococcal disease in CPRD or an International Classification of Diseases (ICD)-10 code (supplementary appendix) for meningococcal disease as the primary discharge diagnosis in the HES databases and an ICD-10 code for any mention of meningococcal disease as the causes of death in the ONS mortality database. The control group included patients identified in the CPRD, HES, and ONS databases without any records of meningococcal disease from January 1, 2008 to December 31, 2017. The meningococcal-related sequelae were assessed during the follow-up period using specific Read codes (supplementary appendix) and ICD-10 codes selected after review of the literature and categorized as per Table 1.

\section{Study outcomes}

Primary outcomes included overall incidence rate of meningococcal disease per 100,000 person-years assessed by year, age group, and diagnosis setting hospital, emergency, outpatient, primary consultation and CFR (by year and age group). Age stratification included the age groups $<1$ year, 1 to 4 years, 5 to 14 years, 15 to 24 years, 25 to 49 years, 50 to 64 years, and $\geq 65$ years. Additional outcomes included descriptive statistics for demographic characteristics, Charlson comorbidity index (CCl) score, high risk status (immunosuppression, active and passive smoking, and winter infections caused by respiratory syncytial virus, influenza, influenza like illness and pneumonia), deaths, and sequelae (supplementary appendix).

\section{Statistical analyses}

Each study measure was summarized using unadjusted methods. Continuous measures were summarised by their medians and the interquartile range (IQR), along with their mean and respective standard deviation (SD). Categorical variables were summarized by numbers and proportions. The annual incidence and CFR due to meningococcal disease for each year of the study (2008-2017) were calculated per 100,000 person-years with the corresponding $95 \%$ confidence interval $(95 \% \mathrm{Cl})$ using the Poisson distribution. The rates for the entire 10 -year study period were calculated as the average of the annual rates between 2008 and 2017. Demographic characteristics of patients with meningococcal disease were described at index date and at the end of follow-up period. For the assessment of disease sequelae, analyses were performed overall and by age group in both cases and matched controls. Descriptive statistics was provided for the analysis of sequelae (at least one sequelae) and by type of sequelae. Incidence risks, incidence rates, time between the index date of meningococcal disease and the occurrence of the first sequelae (time-to-event), were calculated. The incidence risks have been assessed as the number of patients with the sequelae of interest divided by the total number of patients at each time-point. The incidence rate was calculated as the number of first occurrences of each type of sequela during the follow-up period divided by the total aggregate person-time accrued by patients. Kaplan-Meier curves were depicted for the occurrence of sequelae. The $P$-value of log-rank test was computed to compare the survival distributions of cases and controls. Multivariate Cox models were used to adjust the hazard ratio (HR) of sequelae occurrence between cases and controls. Covariates included in the multivariate models were baseline demographic characteristics, $\mathrm{CCl}$ score, and the high-risk status.

\section{Results}

\section{Incidence and mortality}

The study included 640 IMD patients (median age: 7 years [range, 0-98 years]; male: $54.4 \%$ ) with a diagnosis of meningococcal disease between 2008 and 2017. Overall, majority of the patients were diagnosed in a hospital setting (76.9\%), but in those 25 to 49 years old, the diagnosis was made equally at the hospital and primary consultation settings. Over the study period, the median age at diagnosis increased, from 1 year in 2008 to 23 years in 2017 . Analysis by age group showed a decrease in the occurrence of the disease in those aged $<1$ year $(30.4-7.5 \%)$ and an increase in the occurrence of the disease in those $>50$ years (10.4-27.8\%) (Table 2). During the study period, 45 patients died with a mention of meningococcal disease as cause. Mortality rate was slightly higher among females ( $55.6 \%$ vs $44.4 \%)$, toddlers (22.2\%), and adults above 50 years of age (55.6\%).

The incidence of meningococcal disease was higher in the beginning of study period compared with the end, with a decreasing trend over the years (Fig. 2). Annual incidence rates were the highest among those less than 4 years of age, but the incidence rate in these age groups decreased over the study period (from 114.62/100,000 person-years in 2008 to $18.37 / 100,000$ person-years in 2017 in those $<1$ year old and from 33.07/100,000 person-years in 2008 to $5.97 / 100,000$ person-years in 2017 in those 1-4 years old). In adolescents (15-24 years), the disease incidence increased over the study period (from 1.76/100,000 person-years in 2008 to 2.91/100,000 person-years in 2017) with a peak in 2015 and 2016 (4.48 and 4.39/100,000 person-years, respectively).

There were no significant changes in CFR over the study years (CFR $=6.4 \%$ [95\% Cl, 3.6-11] in 2008 and 5.6\% [95\% Cl, 1.2-21.5] in 2017). The highest CFR was reported in 2014 (CFR $=11.6 \%$ [95\% Cl, 5.8-22]), in those 50 years and above of age, ranging from 9.1\% (2016) to 36.4\% (2012). CFR was lower across 
the age groups (<1 year, 5-14 years, 15-24 years, and 25-49 years) compared with that in patients $\geq 50$ years (Fig. 3).

\section{Occurrence of Sequelae (case-control study)}

In total, 552 cases and 2208 controls with a mean follow-up time of $3.3 \pm 2.7$ years were included inthe matched case-control part of this study(Fig. 4). Demographic characteristics of the matched population are displayed in Table 3 . The severity of comorbid diseases was recorded and scored according to the $\mathrm{CCl}$. Cases had a significantly higher frequency of a history of myocardial infarction $(P=0.026)$, congestive heart failure $(P=0.014)$, cerebrovascular disease $(P=0.037)$, pulmonary disease $(P=0.001)$, renal disease $(P=0.002)$, and cancer $(P=0.01)$ (Table 4). When considering CCl category at baseline, cases and controls had similar levels of comorbidities severity for all age groups, except in those $>50$ years old, where cases had significantly more severe comorbidities than controls. When considering all age categories together, cases had significantly more severe comorbidities than controls, but this was mostly driven by those $>50$ years old (data not shown).

During the follow-up period, for all age groups, cases had a higher probability of experiencing at least one sequela than controls (HR, 2.1; $P<0.001)($ Table 5). In total, $61(11.1 \%)$ cases died during the follow-up period. The overall probability of dying was significantly higher in cases than controls, mainly for those above 25 years of age. Except for infants, the probability of having a neurological sequela was consistently higher among cases than controls (HR, $2.39 ; P<$ 0.001). A higher probability of having a physical sequela was observed in cases than controls (HR, 1.63). Higher probability of developing renal conditions in infants and toddlers and cardiovascular conditions in young adults, was observed among cases compared with controls. A higher risk of

psychological/behavioural sequelae was observed among cases than controls, but the difference was not statistically significant (HR, 1.46; $P=0.116)($ Fig. 5). Psychological sequelae category took the longest time to develop with a median of 15.5 months in cases, and as high as 36.2 months in those $<1$ year old; it was followed by neurological sequelae (median, 8.5 months in cases) and physical sequelae (median, 1 month in cases). The risk increased with CCl score and was more than three times higher in those with the highest baseline scores (Fig 6).

\section{Discussion}

This study describes the epidemiology of meningococcal disease and the sequelae associated with meningococcal disease in the UK population using the CPRD database linked to HES and ONS data. The CPRD data have been extensively used for observational research, as it represents $7 \%$ of the UK population, and patients are broadly representative of the general population in terms of age, sex, and ethnicity [17].

The 10-year average annual incidence of meningococcal disease across all age groups in the study was approximately $2.7 / 100,000$ population and decreased over the study period, which is consistent with the data published by Public Health England (PHE) in 2019 [18]. The incidence of the disease was higher in the beginning of our study, in infants and toddlers, and after 2012, no deaths were observed in these age groups. Although discrimination between serogroups was not possible, our data seemed to capture the impact of vaccination against MenC (introduced in 1999) and MenB (introduced in 2015) in the overall number of cases and deaths in the UK [19]. In adolescents (range: 15-24 years), the disease incidence increased over the study period (1.76 per 100,000 in 2008 to 2.91 per 100,000 in 2017) with a peak in 2015 and 2016 (4.48 and 4.39/100,000 person-years, respectively). The decline in the incidence for this age group after 2016 could be explained by the introduction of the quadrivalent ACWY conjugate vaccines into the routine immunization schedule for adolescents in the UK, which took place in 2015 [20].

In our study, the risk of developing at least one sequela was almost double among cases than controls (43.8\% vs $23.1 \%$, [HR, 2.1; $P<0.001])$, which is aligned with published literature [2, 21], and was mainly age-dependent. The most frequent sequelae registered were neurological (21\%), physical (21\%), and/or psychological (5\%). As follow-up durations may differ between studies comparisons should be made with caution.

The study presents some limitations inherent to the nature of the data extracted from the linked CPRD/HES/ONS databases, namely that data were not collected to address our particular research questions, and important variables, such as the vaccination status of the cases or serogroup distribution, were not available for research. The quality of the data on the long-term sequelae depends upon the retention rate of the subjects in the CPRD/HES database, duration of follow-up for each subject, and healthcare resources being tracked in those databases. We cannot exclude that some patients seek care outside of GP practices or hospitals captured in the assessed databases, which could have underestimated the number of cases with sequelae. Moreover, in our study cases had more comorbidities at baseline than controls, hence it is possible that the sequelae were caused due to comorbidities (for example, heart diseases) instead of IMD. Also, pre-existing conditions for the development of sequelae were not assessed, which might have underestimated some complications linked to exacerbation of some pre-existing conditions. In addition, as recognised by the World Health Organization (WHO) "defeating meningitis by 2030 roadmap" [22], there are limited data on the long-term impact of meningitis, and there is limited guidance on how to develop and conduct studies and surveys of sequelae, including its definitions.

\section{Conclusions}

Our findings show that meningococcal disease still poses a significant burden in the UK with patients at an increased risk of developing sequelae which may be associated with additional social and economic burden. The data shows that the analysis of existing data (secondary use of data) could be a useful resource to complement data from the notification systems to better assess the true burden of the disease. Strengthening the prevention through optimisation of vaccination programs may assist in reducing the disease burden. Continuous monitoring of the disease remains an important tool in the prevention and control of this disease and will help in the evaluation of the immunization programs. It is of utmost importance that the monitoring of sequelae is an integral part of the surveillance of meningococcal disease. It should be noted nevertheless that the longitudinal follow-up of patients and the availability of data from different datasets can pose challenges. There is a need for better access to large healthcare databases and development of linkage methods at national level to help characterize the long-term sequelae that meningococcal disease can cause. 


\section{Abbreviations}

CCl: Charlson comorbidities index

CFR: case fatality rate

Cl: confidence interval

CPRD: UK Clinical Practice Research Datalink

DALY: Disability-adjusted life years

HES: Hospital Episode Statistics

HR: hazard ratio

ICD: International Classification of Diseases

IMD: Invasive meningococcal disease

IQR: interquartile range

Men: Meningococcal

ONS: Office for National Statistics

PHE: Public Health England

SD: Standard deviation

UK: United Kingdom

WHO: World Health Organization

YLD: years of life lived with disability

\section{Declarations}

\section{Ethics approval and consent to participate}

The Clinical Practice Research Datalink (CPRD) is an ongoing primary care database of anonymised medical records from general practitioners, with coverage of over 11.3 million patients from 674 practices in the UK. All methods were performed in accordance with the relevant guidelines and regulations and the protocol (reference number 18_203) was approved by the UK CPRD Independent Scientific Advisory Committee (ISAC).

\section{Consent for Publication}

Not applicable.

\section{Availability of data and materials}

The data used for this study were obtained from the Clinical Practice Research Datalink (CPRD). The datasets generated during and/or analysed during the current study are not publicly available but are available from the corresponding author on a reasonable request.

\section{Competing interests}

All authors are employees of Sanofi Pasteur and may hold company shares.

\section{Funding}

The study was funded by Sanofi Pasteur.

\section{Authors' contributions}

All authors have read and approved the manuscript. HB and ST conceptualized the study. Data acquisition was done by ST. SG, HB, EL, IBG, and ST was involved in data analysis, drafting publication, critical revision and providing final approvals.

\section{Acknowledgments}

Medical writing support for this manuscript was provided by Rama Mylapuram, Pharm D (Sanofi) and manuscript editorial support was provided by Anirban Sanyal, PhD (Sanofi). 


\section{References}

1. Meningococcal meningitis. February 2018. Available from: https://www.who.int/news-room/fact-sheets/detail/meningococcal-meningitis Accessed on: December 24, 2020.

2. Pace D, Pollard AJ: Meningococcal disease: clinical presentation and sequelae. Vaccine 2012, 30 Suppl 2:B3-9.

3. Borrow R, Alarcon P, Carlos J, Caugant DA, Christensen H, Debbag R, De Wals P, Echaniz-Aviles G, Findlow J, Head C et al: The Global Meningococcal Initiative: global epidemiology, the impact of vaccines on meningococcal disease and the importance of herd protection. Expert Rev Vaccines 2017 , 16(4):313-328.

4. Rouphael NG, Stephens DS: Neisseria meningitidis: biology, microbiology, and epidemiology. Methods Mol Bio/ 2012, 799:1-20.

5. Stephens DS, Greenwood B, Brandtzaeg P: Epidemic meningitis, meningococcaemia, and Neisseria meningitidis. Lancet 2007, 369(9580):2196-2210.

6. Vyse A, Ellsbury G, Madhava H: Protecting UK adolescents and adults against meningococcal serogroup B disease. Expert Rev Vaccines 2018, 17(3):229237.

7. Invasive meningococcal disease. Annual Epidemiological Report for 2017. European Center for Disease Control and Prevention. Available from: https://www.ecdc.europa.eu/sites/default/files/documents/AER_for_2017-invasive-meningococcal-disease.pdf Accessed on: December 24, 2020.

8. Enhanced Meningococcal Disease Surveillance Report, 2018. Confirmed and Probable Cases Reported to the National Notifiable Diseases Surveillance System, 2018. CDC. Available from: https://www.cdc.gov/meningococcal/downloads/NCIRD-EMS-Report-2018.pdf Accessed on: December 24, 2020.

9. Gunaratnam P, Massey P, Durrheim D, Torvaldsen S: Invasive meningococcal disease in elderly people, New South Wales, Australia, 1993 to 2012 . Western Pac Surveill Response J 2013, 4(4):4-10.

10. Trzewikoswki de Lima G, De Gaspari E: Study of the Immune Response in the Elderly: Is It Necessary to Develop a Vaccine against Neisseria meningitidis for the Aged? J Aging Res 2019, 2019:9287121.

11. DALYs GBD, Collaborators H, Murray CJ, Barber RM, Foreman KJ, Abbasoglu Ozgoren A, Abd-Allah F, Abera SF, Aboyans V, Abraham JP et al: Global, regional, and national disability-adjusted life years (DALYs) for 306 diseases and injuries and healthy life expectancy (HALE) for 188 countries, 1990 2013: quantifying the epidemiological transition. Lancet 2015, 386(10009):2145-2191.

12. Collaborators GBDM: Global, regional, and national burden of meningitis, 1990-2016: a systematic analysis for the Global Burden of Disease Study 2016. Lancet Neurol 2018, 17(12):1061-1082.

13. Ladhani SN, Flood JS, Ramsay ME, Campbell H, Gray SJ, Kaczmarski EB, Mallard RH, Guiver M, Newbold LS, Borrow R: Invasive meningococcal disease in England and Wales: implications for the introduction of new vaccines. Vaccine 2012, 30(24):3710-3716.

14. Safadi MA, McIntosh ED: Epidemiology and prevention of meningococcal disease: a critical appraisal of vaccine policies. Expert Rev Vaccines 2011, 10(12):1717-1730.

15. Stefanelli P, Rezza G: Impact of vaccination on meningococcal epidemiology. Hum Vaccin Immunother 2016, 12(4):1051-1055.

16. Gobin M, Hughes G, Foulkes S, Bagnall H, Trindall A, Decraene V, Edeghere O, Balasegaram S, Cummins A, Coole L: The epidemiology and management of clusters of invasive meningococcal disease in England, 2010-15. J Public Health (Oxf) 2020, 42(1):e58-e65.

17. Herrett E, Gallagher AM, Bhaskaran K, Forbes H, Mathur R, van Staa T, Smeeth L: Data Resource Profile: Clinical Practice Research Datalink (CPRD). Int J Epidemio/ 2015, 44(3):827-836.

18. Invasive meningococcal disease in England: annual laboratory confirmed reports for epidemiological year 2018/2019. Health Protection Report Volume 13 Number 38. Available from:

https://assets.publishing.service.gov.uk/government/uploads/system/uploads/attachment_data/file/842368/hpr3819_IMD-ann.pdf Accessed on: 28 December 2020.

19. Parikh SR, Campbell H, Bettinger JA, Harrison LH, Marshall HS, Martinon-Torres F, Safadi MA, Shao Z, Zhu B, von Gottberg A et al: The everchanging epidemiology of meningococcal disease worldwide and the potential for prevention through vaccination. $J$ Infect 2020, 81(4):483-498.

20. Public Health England. The Green Book. Chapter 22: Meningococcal. Published Mar 2012. Last updated Sept 2016. Available from: https://www.gov.uk/government/publications/meningococcal-the-green-book-chapter-22 Accessed on: 28 Decemeber 2020.

21. Huang L, Heuer OD, Janssen S, HackI D, Schmedt N: Clinical and economic burden of invasive meningococcal disease: Evidence from a large German claims database. PLoS One 2020, 15(1):e0228020.

22. WHO. Defeating meningitis by 2030: a global road map. Available at: https://www.who.int/publications/m/item/defeating-meningitis-by-2030-a-globalroad-map Accessed on: 31Jan2021

\section{Tables}

Table 1: Sequelae categories 


\begin{tabular}{|c|c|c|}
\hline Categories & Sub-categories & Types \\
\hline \multirow[t]{5}{*}{ Physical } & Dermatological conditions & Skin scarring (including skin graft) \\
\hline & Cardiovascular conditions & $\begin{array}{l}\text { Symptoms consistent with Raynaud phenomenon, venous thrombosis, vasculitis, pericarditis, } \\
\text { endocarditis, pericardiocentesis, and cardiac arrest }\end{array}$ \\
\hline & Renal conditions & Renal failure (acute and chronic) and urinary failure \\
\hline & $\begin{array}{l}\text { Musculoskeletal deficiencies (bone, } \\
\text { joint, muscle) }\end{array}$ & Arthritis, limb deficiency/deformities, amputation, arthralgia, and bone growth distortion \\
\hline & Other physical conditions & $\begin{array}{l}\text { Pulmonary condition, respiratory distress syndrome, sepsis, toxic shock syndrome, disseminated } \\
\text { intravascular coagulation, coma, gangrene, diabetes insipidus, acute liver disease, sequelae of } \\
\text { other specified infectious and parasitic diseases, and disorder of tooth development }\end{array}$ \\
\hline \multirow[t]{6}{*}{ Neurological } & Sensory system deficits & Blindness and hearing loss (mild, moderate, severe, and profound) \\
\hline & Motor deficits & $\begin{array}{l}\text { Paralysis, cerebral palsies, muscle weakness, monoparesis, hemiparesis, movement coordination, } \\
\text { spasticity, mobility problems, severe neuromotor-impairment, and balance impairment }\end{array}$ \\
\hline & Communications disorders & Aphasia, general speech, and language and communication difficulties \\
\hline & Intellectual disability & Mental retardation (IQ < 70), mild IQ loss (IQ 70-85), learning disabilities, and cognitive deficits \\
\hline & Abnormal brain activity & $\begin{array}{l}\text { Seizures (epileptic and non-epileptic), chronic headaches/migraine, dizziness and giddiness, and } \\
\text { disorders of vestibular function }\end{array}$ \\
\hline & Other severe neurological disorders & Hydrocephalus \\
\hline \multirow[t]{3}{*}{ Psychological/behavioural } & Anxiety disorders & Generalized anxiety, separation anxiety, social anxiety disorder, and specific phobia \\
\hline & Behavioural disorders & Conduct disorder \\
\hline & $\begin{array}{l}\text { Other } \\
\text { psychological/emotional/behavioural } \\
\text { disorders }\end{array}$ & $\begin{array}{l}\text { Depression, post-traumatic stress disorder, disturbance of activity and attention, and other } \\
\text { disorders of psychological development }\end{array}$ \\
\hline
\end{tabular}

Abbreviation: IQ, intelligence quotient.

Table 2: Demographic characteristics of patients with meningococcal disease 


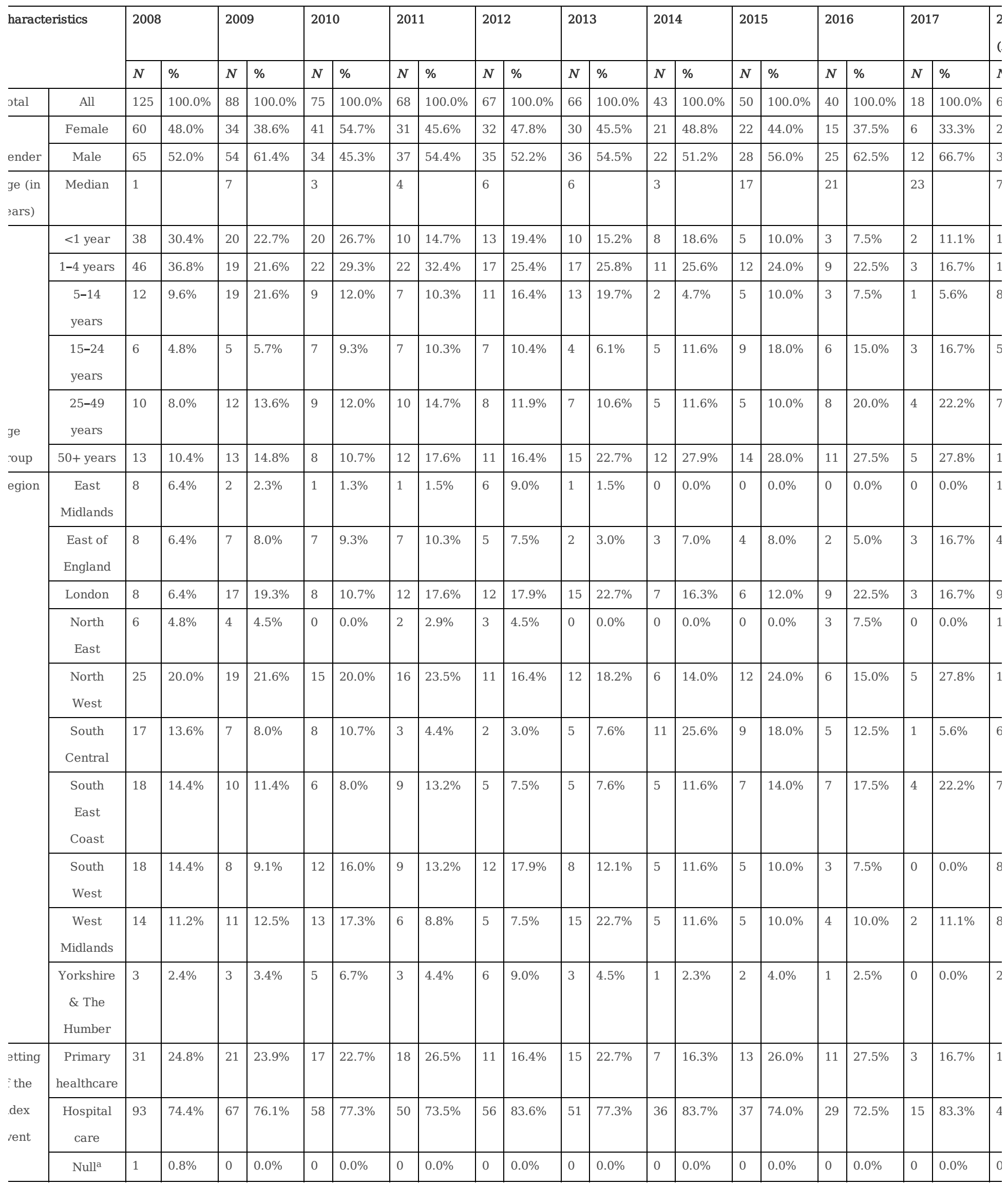

a One patient diagnosed with only meningococcal disease was recorded in mortality data.

Table 3: Demographic characteristics of patients in the case-control study 


\begin{tabular}{|c|c|c|c|c|c|}
\hline \multirow[b]{2}{*}{ Characteristics } & & \multicolumn{2}{|c|}{ Case } & \multicolumn{2}{|c|}{ Control } \\
\hline & & $N$ & $\%$ & $N$ & $\%$ \\
\hline Total & Total & 552 & $100.0 \%$ & 2208 & $100.0 \%$ \\
\hline \multirow[t]{2}{*}{ Gender } & Female & 253 & $45.8 \%$ & 1012 & $45.8 \%$ \\
\hline & Male & 299 & $54.2 \%$ & 1196 & $54.2 \%$ \\
\hline \multirow[t]{2}{*}{ Age at index date (in years) } & Average & 19.95 & & 19.95 & \\
\hline & Standard deviation & 26.16 & & 26.15 & \\
\hline \multirow[t]{6}{*}{ Age group } & $<1$ year & 127 & $23.0 \%$ & 508 & $23.0 \%$ \\
\hline & $1-4$ years & 141 & $25.5 \%$ & 564 & $25.5 \%$ \\
\hline & 5-14 years & 74 & $13.4 \%$ & 296 & $13.4 \%$ \\
\hline & 15-24 years & 50 & $9.1 \%$ & 200 & $9.1 \%$ \\
\hline & 25-49 years & 62 & $11.2 \%$ & 248 & $11.2 \%$ \\
\hline & $50+$ years & 98 & $17.8 \%$ & 392 & $17.8 \%$ \\
\hline \multirow[t]{10}{*}{ Region } & East Midlands & 16 & $2.9 \%$ & 64 & $2.9 \%$ \\
\hline & East of England & 47 & $8.5 \%$ & 188 & $8.5 \%$ \\
\hline & London & 86 & $15.6 \%$ & 344 & $15.6 \%$ \\
\hline & North East & 12 & $2.2 \%$ & 48 & $2.2 \%$ \\
\hline & North West & 115 & $20.8 \%$ & 460 & $20.8 \%$ \\
\hline & South Central & 55 & $10.0 \%$ & 220 & $10.0 \%$ \\
\hline & South East Coast & 63 & $11.4 \%$ & 252 & $11.4 \%$ \\
\hline & South West & 67 & $12.1 \%$ & 268 & $12.1 \%$ \\
\hline & West Midlands & 70 & $12.7 \%$ & 280 & $12.7 \%$ \\
\hline & Yorkshire and the Humber & 21 & $3.8 \%$ & 84 & $3.8 \%$ \\
\hline \multirow[t]{8}{*}{ Race } & Missing & 14 & $2.5 \%$ & 294 & $13.3 \%$ \\
\hline & Black African & 3 & $0.5 \%$ & 27 & $1.2 \%$ \\
\hline & Black Caribbean & 3 & $0.5 \%$ & 12 & $0.5 \%$ \\
\hline & Black other & 3 & $0.5 \%$ & 8 & $0.4 \%$ \\
\hline & Indian, Pakistan, and Bangladeshi & 9 & $1.6 \%$ & 69 & $3.1 \%$ \\
\hline & Other and mixed & 23 & $4.2 \%$ & 76 & $3.4 \%$ \\
\hline & Other Asian & 6 & $1.1 \%$ & 38 & $1.7 \%$ \\
\hline & White & 491 & $88.9 \%$ & 1684 & $76.3 \%$ \\
\hline
\end{tabular}

Abbreviation: $N$, number.

\section{Table 4: Charlson comorbidities at baseline (case-control study)}




\begin{tabular}{|l|l|l|l|l|l|}
\hline \multirow{2}{*}{ Charlson comorbidities } & \multicolumn{2}{l|l}{ Cases } & \multicolumn{2}{ll}{ Controls } & \multirow{2}{*}{-value $^{\mathrm{a}}$} \\
\cline { 2 - 5 } & $\boldsymbol{N}$ & $\%$ & $\boldsymbol{N}$ & $\%$ & \\
\hline$N$ & 552 & & 2208 & & \\
\hline Myocardial infarction & 5 & $0.9 \%$ & 5 & $0.2 \%$ & 0.026 \\
\hline Congestive heart failure & 7 & $1.3 \%$ & 8 & $0.4 \%$ & 0.014 \\
\hline Peripheral vascular disease & 4 & $0.7 \%$ & 9 & $0.4 \%$ & 0.338 \\
\hline Cerebrovascular disease & 7 & $1.3 \%$ & 10 & $0.5 \%$ & 0.037 \\
\hline Dementia & 2 & $0.4 \%$ & 12 & $0.5 \%$ & 0.582 \\
\hline Pulmonary disease & 36 & $6.5 \%$ & 78 & $3.5 \%$ & 0.001 \\
\hline Connective tissue disorder & 3 & $0.5 \%$ & 6 & $0.3 \%$ & 0.327 \\
\hline Peptic ulcer disease & 0 & $0.0 \%$ & 0 & $0.0 \%$ & NA \\
\hline Mild liver disease & 3 & $0.5 \%$ & 2 & $0.1 \%$ & 0.05 \\
\hline Diabetes without complications & 20 & $3.6 \%$ & 26 & $1.2 \%$ & $<0.001$ \\
\hline Diabetes with complications & 0 & $0.0 \%$ & 3 & $0.1 \%$ & 0.992 \\
\hline Paraplegia & 2 & $0.4 \%$ & 2 & $0.1 \%$ & 0.166 \\
\hline Renal disease & 11 & $2.0 \%$ & 13 & $0.6 \%$ & 0.002 \\
\hline Cancer & 2 & $2.2 \%$ & 20 & $0.9 \%$ & 0.01 \\
\hline Moderate or severe liver disease & 2 & $0.4 \%$ & 0 & $0.0 \%$ & 0.992 \\
\hline Metastatic cancer & $0.2 \%$ & 7 & $0.3 \%$ & 0.589 \\
\hline AIDS/HIV & 3 & 0 & $0.0 \%$ & \multirow{2}{*}{ NA } \\
\hline
\end{tabular}

\begin{tabular}{|c|c|c|c|c|c|c|}
\hline & & \multicolumn{2}{|c|}{ Cases } & \multicolumn{2}{|c|}{ Controls } & \multirow[t]{2}{*}{$P$-value } \\
\hline \multicolumn{2}{|c|}{ Charlson comorbidity index } & $N$ & $\%$ & $N$ & $\%$ & \\
\hline$N$ & Total & 552 & & 2208 & & $<0.001^{\mathrm{b}}$ \\
\hline \multirow[t]{2}{*}{ CCI score } & Average & 0.27 & & 0.12 & & \\
\hline & Standard deviation & 0.88 & & 0.63 & & \\
\hline \multirow[t]{4}{*}{ CCI category } & 0 & 481 & $87.1 \%$ & 2057 & $93.2 \%$ & $<0.001^{\mathrm{c}}$ \\
\hline & $1-2$ & 50 & $9.1 \%$ & 126 & $5.7 \%$ & \\
\hline & $3-4$ & 17 & $3.1 \%$ & 15 & $0.7 \%$ & \\
\hline & $\geq 5$ & 4 & $0.7 \%$ & 10 & $0.5 \%$ & \\
\hline
\end{tabular}

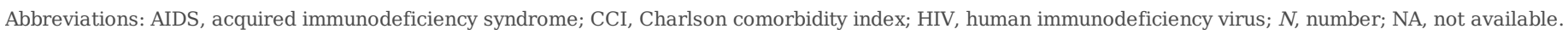
$\mathrm{a}_{\text {Univariate conditional logistic regression. }}$

$\mathrm{b}_{\text {Wilcoxon's test. }}$

$\mathrm{C}_{\text {Univariate conditional logistic regression. }}$

Table 5: Sequelae observed during follow-up 


\begin{tabular}{|c|c|c|c|c|c|c|}
\hline \multirow[t]{2}{*}{ Sequelae } & \multicolumn{3}{|c|}{ Case $(N=552)$} & \multicolumn{3}{|c|}{ Control $(N=2208)$} \\
\hline & $N$ & Risk (\%) & Rate (/1000 PY) & $N$ & Risk (\%) & Rate (/1000 PY) \\
\hline At least one complication & 242 & $43.8(39.8-48.0)$ & $191.4(168.1-217.1)$ & 510 & $23.1(21.4-24.9)$ & $82.7(75.7-90.2)$ \\
\hline Death & 61 & $11.1(8.7-13.9)$ & $33.3(25.5-42.8)$ & 93 & $4.2(3.5-5.1)$ & $12.6(10.2-15.5)$ \\
\hline Neurological sequelae & 116 & $21.0(17.8-24.6)$ & $77.2(63.8-92.6)$ & 208 & $9.4(8.3-10.7)$ & $30.8(26.7-35.3)$ \\
\hline Abnormal brain activity & 88 & $15.9(13.1-19.2)$ & $55.8(44.8-68.8)$ & 140 & $6.3(5.4-7.4)$ & $20.2(17.0-23.9)$ \\
\hline Communication disorder & 4 & $0.7(0.3-1.8)$ & $2.2(0.6-5.7)$ & 9 & $0.4(0.2-0.8)$ & $1.2(0.6-2.4)$ \\
\hline Intellectual disability & 19 & $3.4(2.2-5.3)$ & $10.6(6.4-16.6)$ & 46 & $2.1(1.6-2.8)$ & $6.4(4.7-8.5)$ \\
\hline Motor deficits & 0 & $0.0(0.0-0.7)$ & $0.0(0.0-2.0)$ & 1 & $0.0(0.0-0.3)$ & $0.1(0.0-0.8)$ \\
\hline Sensory system deficits & 14 & $2.5(1.5-4.2)$ & $7.9(4.3-13.2)$ & 28 & $1.3(0.9-1.8)$ & $3.9(2.6-5.6)$ \\
\hline Other neurological complications & 3 & $0.5(0.2-1.6)$ & $1.7(0.3-4.9)$ & 2 & $0.1(0.0-0.3)$ & $0.3(0.0-1.0)$ \\
\hline Physical sequelae & 115 & $20.8(17.7-24.4)$ & $76.8(63.4-92.2)$ & 268 & $12.1(10.8-13.6)$ & $40.7(36.0-45.9)$ \\
\hline Cardio/vascular conditions & 10 & $1.8(1.0-3.3)$ & $5.6(2.7-10.2)$ & 16 & $0.7(0.4-1.2)$ & $2.2(1.3-3.6)$ \\
\hline Dermatological conditions & 9 & $1.6(0.9-3.1)$ & $5.0(2.3-9.5)$ & 33 & $1.5(1.1-2.1)$ & $4.6(3.2-6.5)$ \\
\hline Musculoskeletal deficiencies & 22 & $4.0(2.6-6.0)$ & $12.6(7.9-19.0)$ & 64 & $2.9(2.3-3.7)$ & $9.0(6.9-11.5)$ \\
\hline Renal conditions & 34 & $6.2(4.4-8.5)$ & $19.3(13.4-27.0)$ & 64 & $2.9(2.3-3.7)$ & $8.9(6.8-11.3)$ \\
\hline Skin scarring & 0 & $0.0(0.0-0.7)$ & $0.0(0.0-2.0)$ & 0 & $0.0(0.0-0.2)$ & $0.0(0.0-0.5)$ \\
\hline Other physical conditions & 78 & $14.1(11.5-17.3)$ & $49.4(39.0-61.6)$ & 135 & $6.1(5.2-7.2)$ & $19.5(16.4-23.1)$ \\
\hline Psychological/behavioural sequelae & 26 & $4.7(3.2-6.8)$ & $15.0(9.8-21.9)$ & 66 & $3.0(2.4-3.8)$ & $9.3(7.2-11.8)$ \\
\hline Anxiety disorders & 8 & $1.4(0.7-2.8)$ & $4.5(1.9-8.8)$ & 22 & $1.0(0.7-1.5)$ & $3.0(1.9-4.6)$ \\
\hline Behavioural disorders & 0 & $0.0(0.0-0.7)$ & $0.0(0.0-2.0)$ & 0 & $0.0(0.0-0.2)$ & $0.0(0.0-0.5)$ \\
\hline Other psychological/emotional/behavioural disorders & 22 & $4.0(2.6-6.0)$ & $12.5(7.9-19.0)$ & 54 & $2.4(1.9-3.2)$ & $7.5(5.7-9.8)$ \\
\hline
\end{tabular}

Abbreviations: $N$, number; PY, person-years.

\section{Figures}

\section{Figure 1:}

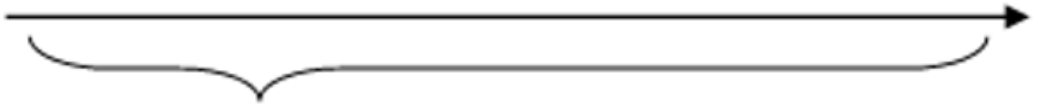

Index date:

\section{Eligibility start}

January 1, 2007
Date of first episode of MD identified by

READ / ICD-10 codes in CPRD, HES, and ONS databases
Eligibility end

December 31, 2017

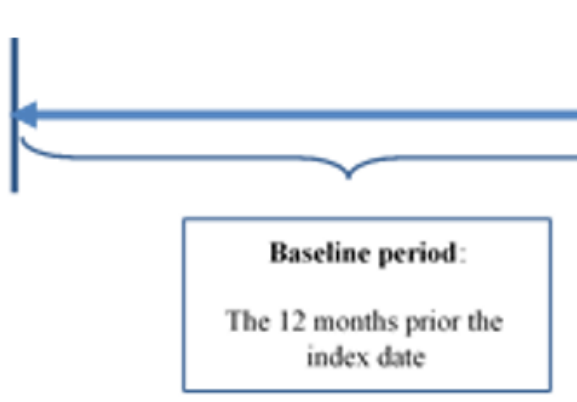

Follow-up period:

All the data available after the index date until the earliest of date of last collection, date of transfer out of the GP practice or the date of death 
Figure 1

Study design. CPRD, UK Clinical Practice Research Datalink; GP, general practitioner; HES, Hospital Episode Statistics; ICD, International Classification of Diseases; MD, meningococcal disease; ONS, Office for National Statistics.

\section{Figure 2:}

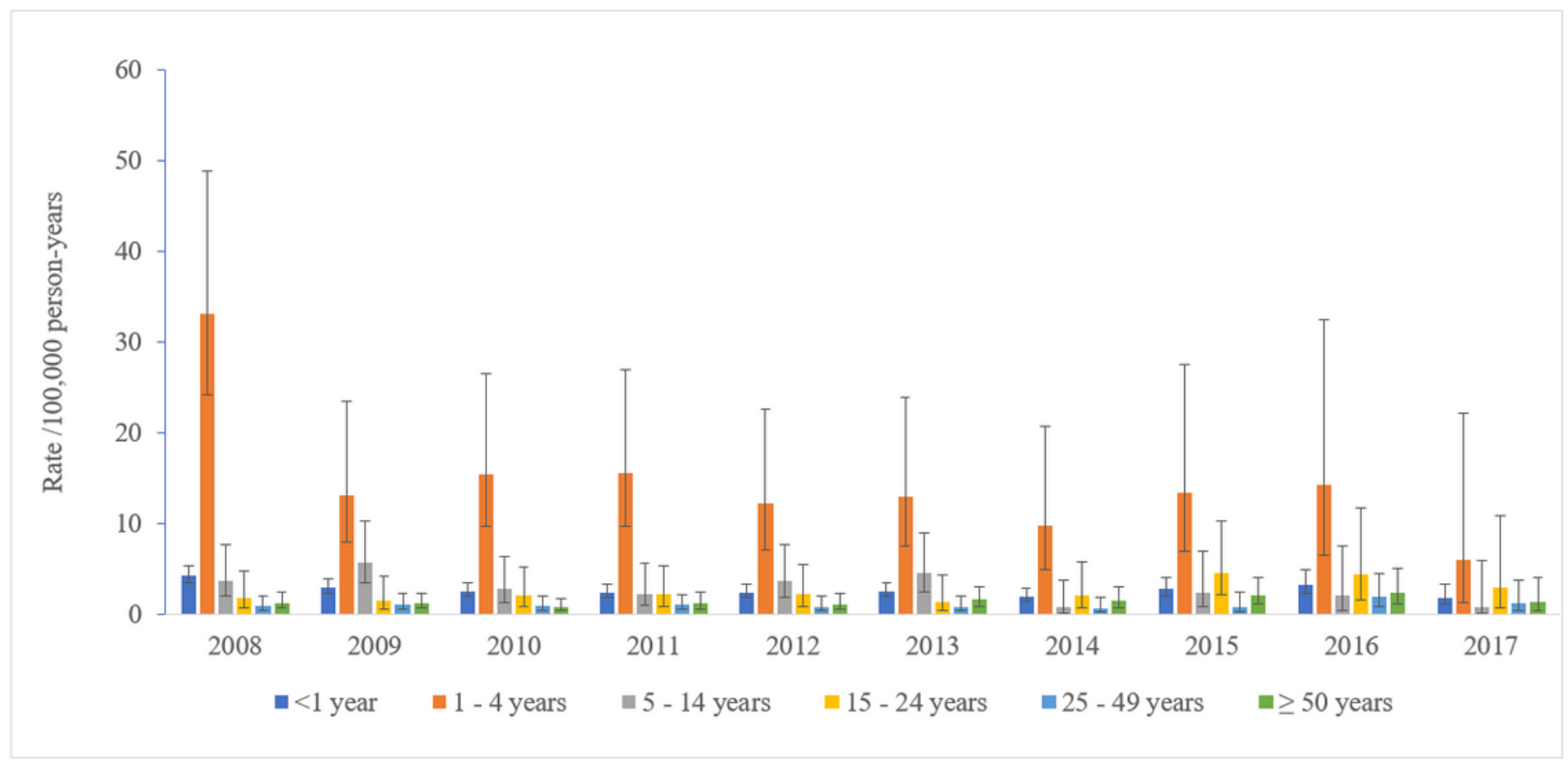

Figure 2

Annual incidence rates of meningococcal disease from 2008 to 2017 by age group

Figure 3:

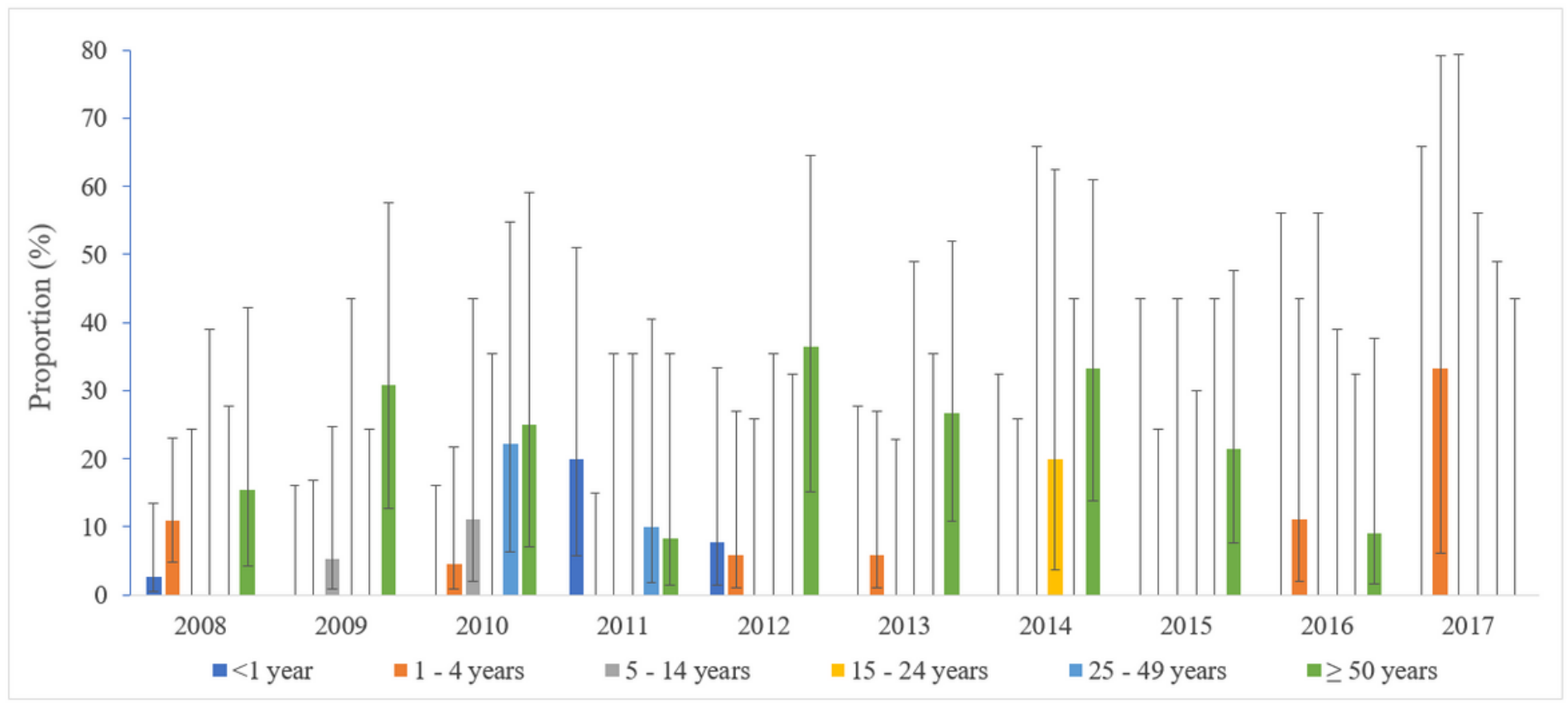

Figure 3

Case fatality rates from 2008 to 2017 by age group

Page 12/15 


\section{Figure 4:}

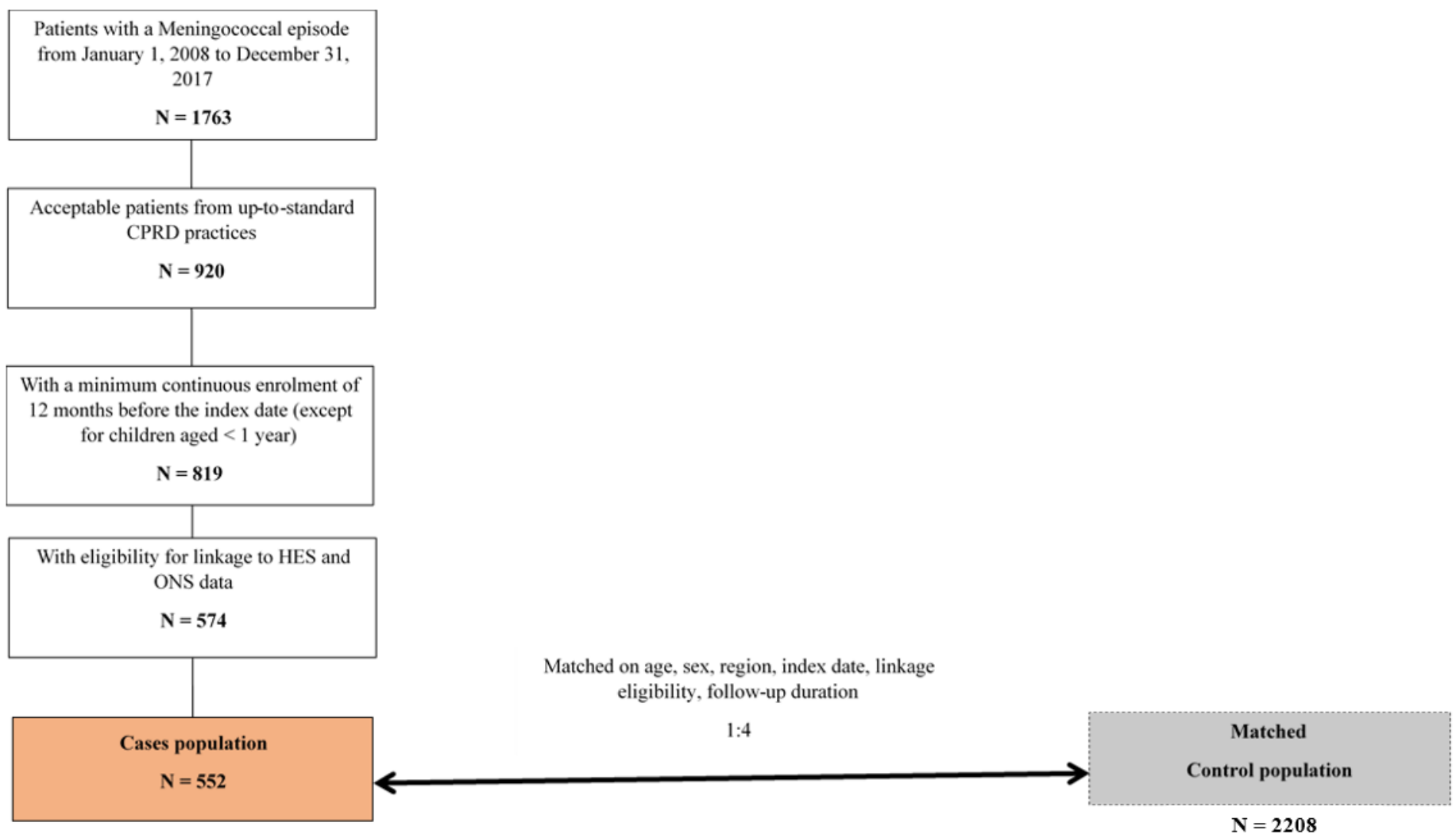

Figure 4

Patient selection (case-control study) 


\section{Figure 5:}
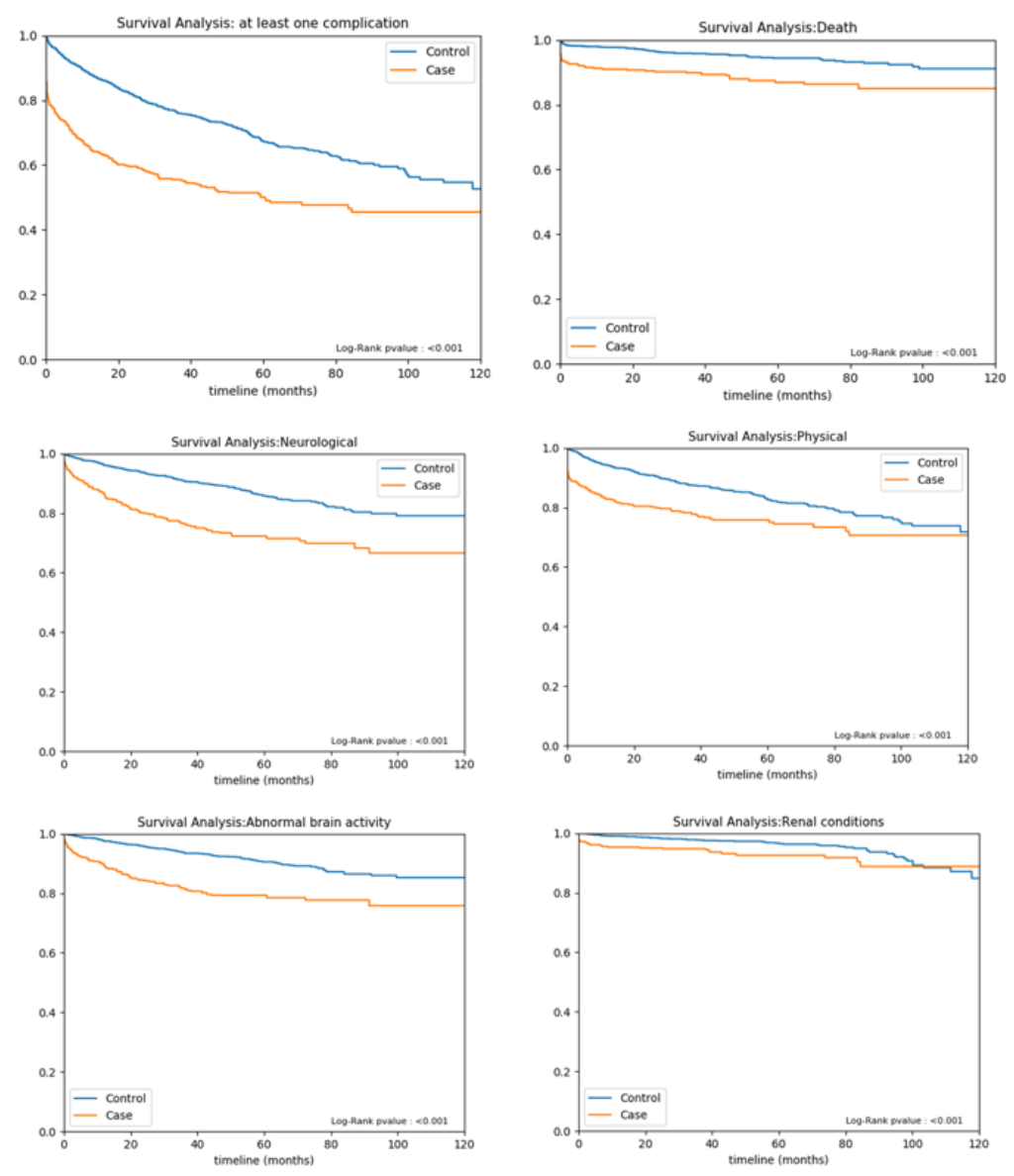

Figure 5

Kaplan-Meier curves of the occurrence of sequelae during the follow-up period 
Figure 6:

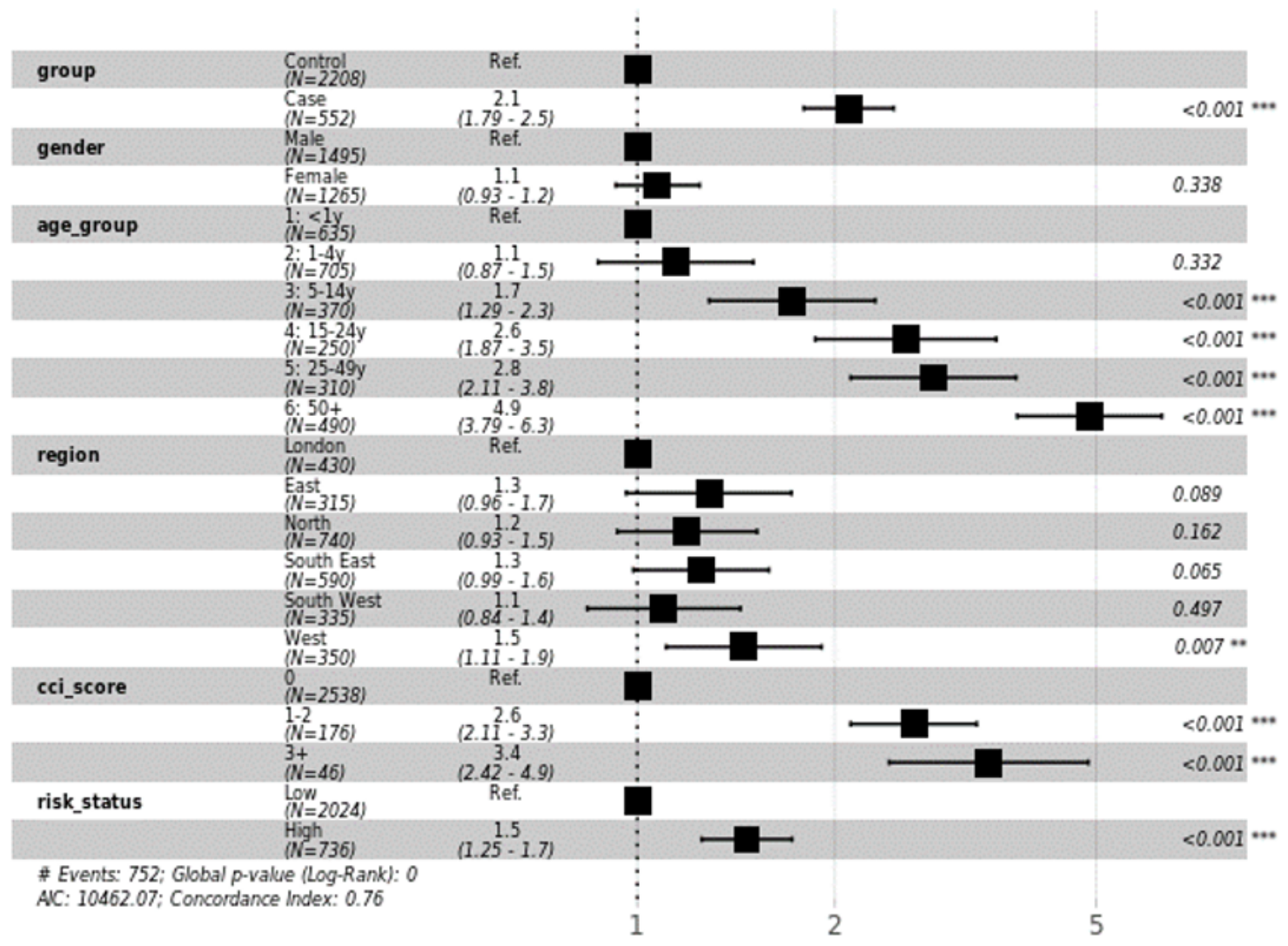

Figure 6

Hazard ratios for sequelae during the follow-up period using the multivariate cox regression model

\section{Supplementary Files}

This is a list of supplementary files associated with this preprint. Click to download.

- Supplementaryappendix.xIsx 